\title{
Rationale and design of the Eplerenone combination Versus conventional Agents to Lower blood pressure on Urinary Antialbuminuric Treatment Effect (EVALUATE) trial: a double-blinded randomized placebo-controlled trial to evaluate the antialbuminuric effects of an aldosterone blocker in hypertensive patients with albuminuria
}

Katsuyuki Ando ${ }^{1}$, Hiroshi Ohtsu ${ }^{2}$, Yoshihiro Arakawa ${ }^{3}$, Kiyoshi Kubota ${ }^{4}$, Takuhiro Yamaguchi ${ }^{2}$, Miki Nagase ${ }^{1}$, Akira Yamada ${ }^{5}$ and Toshiro Fujita ${ }^{1}$, on behalf of the EVALUATE Study Investigators

Although inhibitors of the renin-angiotensin system are effective as first-line antihypertensive drugs in hypertensive patients with chronic kidney disease, they cannot completely prevent the progression of renal injury. Many animal studies, including our own, and a few human studies suggest that mineralocorticoid receptor blockade could inhibit the ongoing renal damage in chronic kidney disease. Thus, we designed this double-blinded, randomized, placebo-controlled trial to evaluate the antialbuminuric effect of a low dose $\left(50 \mathrm{mg} \mathrm{day}^{-1}\right)$ of the mineralocorticoid receptor antagonist eplerenone. The study subjects will include 340 hypertensive patients (blood pressure: $130-180 / 80-100 \mathrm{~mm} \mathrm{Hg}$ ) with albuminuria (urinary albumin/creatinine ratio: $30-600 \mathrm{mg} \mathrm{g}^{-1}$ in the first morning void urine), who are treated with an inhibitor of the renin-angiotensin system. Other classes of antihypertensive drugs may be added as needed to achieve the target blood pressure $(<130 / 80 \mathrm{~mm} \mathrm{Hg})$. The primary study end point is the change in the urinary albumin/creatinine ratio after a 1-year study period. This trial is expected to show whether a low dose of mineralocorticoid receptor antagonists can exert an antialbuminuric effect in patients with chronic kidney disease. Hypertension Research (2010) 33, 616-621; doi:10.1038/hr.2010.46; published online 9 April 2010

Keywords: mineralocorticoid receptor antagonist; urinary albumin; chronic kidney disease

\section{INTRODUCTION}

Chronic kidney disease (CKD) should be treated properly while it is still at an early stage because it is strongly associated with progression to both end-stage kidney disease (ESKD) and cardiovascular disease (CVD). As CKD is often associated with hypertension, which accelerates the progression of both ESKD and CVD, strict blood pressure (BP) control is essential for its management. Thus, CKD patients are usually given antihypertensive drugs. Considerable clinical evidence shows that renin-angiotensin system (RAS) inhibitors, such as angiotensin receptor blockers (ARBs) and angiotensin-converting enzyme (ACE) inhibitors, are acceptable as first-line antihypertensive agents for CKD. ${ }^{1,2,3}$ However, they cannot completely prevent CKD progression. Thus, second-line depressor agents for CKD are needed. Currently, these treatments include calcium channel blockers (CCBs) or diuretics, ${ }^{3}$ but the evidence supporting this approach is weak. ${ }^{4,5}$ More effective renoprotective antihypertensive agents are required. New lines of evidence suggest that mineralocorticoid receptor (MR)-blocking agents may be suitable for this purpose.

Renoprotective effect of MR blockade

Recent studies revealed that MR antagonists have renoprotective effects. Although the infusion of aldosterone and excessive dietary salt progressively induce proteinuria and renal podocyte injury in rats, these effects are almost completely reversed by the MR antagonist

${ }^{1}$ Department of Nephrology and Endocrinology, Faculty of Medicine, University of Tokyo Hospital, Tokyo, Japan; ${ }^{2}$ Department of Clinical Trial Data Management, Faculty of Medicine, University of Tokyo Hospital, Tokyo, Japan; ${ }^{3}$ University of Tokyo Clinical Research Center, University of Tokyo Hospital, University of Tokyo, Tokyo, Japan; ${ }^{4}$ Department of Pharmacoepidemiology, Faculty of Medicine, University of Tokyo, Tokyo, Japan and ${ }^{5}$ First Department of Internal Medicine, Kyorin University School of Medicine, Tokyo, Japan Correspondence: Dr T Fujita, Department of Nephrology and Endocrinology, Faculty of Medicine, University of Tokyo, 7-3-1, Hongo, Bunkyo-ku, Tokyo, 113-8655, Japan. E-mail: fujita-dis@h.u-tokyo.ac.jp

Received 14 January 2010; accepted 9 February 2010; published online 9 April 2010 
eplerenone. ${ }^{6}$ Eplerenone also suppresses the progressive renal injury of the obese spontaneously hypertensive rats (SHR), known as SHR/NDmcr-cp, which exhibit metabolic syndrome characteristics and have high plasma aldosterone levels. ${ }^{7}$ Importantly, eplerenone ameliorates renal injury in a low aldosterone model of CKD as well. ${ }^{8}$ Thus, plasma aldosterone levels do not necessarily seem to reflect renal MR activity. These observations suggest that aldosterone-dependent ${ }^{6,7}$ and/or aldosterone-independent ${ }^{8-11} \mathrm{MR}$ activation may have an essential role in CKD pathophysiology and that MR blockade may be an effective renoprotective strategy. Indeed, clinical studies have shown that plasma aldosterone profiles are not predictive of the antihypertensive efficacy of MR blockade. ${ }^{12}$ Accordingly, the antialbuminuric effect of the MR blocker may also not be predicted by plasma aldosterone concentration.

\section{Renal effects of MR blockade vs. RAS inhibition}

In salt-loaded Dahl salt-sensitive rats, eplerenone suppresses the renal injury more effectively than the ACE inhibitor and to a similar degree as the combination of both drugs. ${ }^{13}$ In addition, in stroke-prone spontaneously hypertensive rats (SHRSP), aldosterone infusion completely abolishes the renoprotective effect of the ACE inhibitor. ${ }^{14}$ Thus, MR blockade may be even better than RAS-inhibiting agents at suppressing renal injury progression. Supporting this hypothesis are data indicating that RAS blockade does not ameliorate diabetic nephropathy in patients with aldosterone breakthrough (where plasma aldosterone levels again increase after long-term RAS-inhibitor treatment), ${ }^{15}$ whereas the aldosterone antagonist, spironolactone, decreases the urinary protein levels of such patients.

\section{Human studies of renoprotection by MR blockade}

Several human studies have revealed that MR antagonists have renoprotective effects in addition to the cardioprotective action that has been already established by clinical mega-studies. ${ }^{16,17}$ For example, eplerenone reduces the urinary albumin/creatinine $(\mathrm{Cr})$ ratios in older patients with systolic hypertension and microalbuminuria much better than the CCB, amlodipine. ${ }^{18}$ Moreover, although both eplerenone and the ACE inhibitor, enalapril, decrease urinary albumin levels in hypertensive patients with albuminuria, eplerenone has a superior antialbuminuric effect. ${ }^{12}$ Although high doses (up to 200 $\mathrm{mg} \mathrm{day}{ }^{-1}$ ) of eplerenone were used in these studies, a short duration (12 weeks) of treatment with relatively low doses $\left(50-100 \mathrm{mg} \mathrm{day}^{-1}\right.$ ) of eplerenone has also been shown to decrease urinary albumin/Cr ratios in a small number of RAS inhibitor-treated patients with type 2 diabetic nephropathy. ${ }^{19}$ Thus, a long-term and larger trial testing whether a low dose of eplerenone can protect the renal function of CKD patients is necessary.

\section{Renoprotective effect of MR blockade in Japanese patients}

Aldosterone induces inflammatory cell infiltration and vasculitis in the kidneys of rats in the context of excessive salt intake. ${ }^{20,21}$ We also observed that aldosterone treatment causes proteinuria and podocyte injury in rats fed a high salt diet. ${ }^{6}$ Furthermore, in subjects with high urinary aldosterone levels, urinary protein levels increase progressively as dietary salt levels rise. ${ }^{22}$ Thus, the harmful effects of aldosterone on the renocardiovascular system become apparent on salt repletion. ${ }^{23,24}$ As salt intake in Japan is high on average, ${ }^{25}$ it may be useful to treat Japanese CKD patients with MR blockers.

\section{Evaluation of the antialbuminuric effect of eplerenone}

It is, therefore, of considerable interest to determine whether a low dose $\left(50 \mathrm{mg} \mathrm{day}^{-1}\right)$ of the highly MR-specific antagonist, eplerenone, has antialbuminuric effects in hypertensive CKD patients in Japan. Consequently, we designed the double-blinded, randomized, placebocontrolled Eplerenone combination Versus conventional Agents to Lower blood pressure on Urinary Antialbuminuric Treatment Effect (EVALUATE) trial to compare the antialbuminuric effect between a 50-mg day ${ }^{-1}$ eplerenone dose and placebo.

Hypothesis and objectives. Our hypothesis is that the MR blockade induced by a low dose of eplerenone effectively suppresses renal injury progression in RAS inhibitor-treated CKD patients. The primary trial objective is to compare the antialbuminuric effects of a 1-year treatment with eplerenone or placebo. Key secondary objectives are: (1) to compare eplerenone- and placebo-treated patients in terms of changes in serum $\mathrm{Cr}$ levels, estimated glomerular filtration rate (eGFR) and urinary liver-type free fatty acid-binding protein levels (L-FABP; an early marker of tubulointerstitial stress) ${ }^{26,27}$ and (2) to determine the effect of high salt intake on the putative renoprotective effects of eplerenone.

\section{METHODS}

\section{End points}

Primary end point. This end point refers to the percent change in the urinary albumin/Cr ratio in the first morning void urine after 12 treatment months relative to the pretreatment ratio (an average of three continuously measured values). Urinary albumin and $\mathrm{Cr}$ levels will be measured by immunoturbidimetrical (autoanalyzer: JCA-BM8000 series, JEOL, Tokyo, Japan) and enzymatic (JCA-BM8000 series, JEOL) methods, respectively.

Secondary end points. These end points are the absolute values and percent changes at each treatment period relative to the pretreatment values of urinary albumin/Cr ratio $(4,8,28$ and 52 weeks after the start of the trial drugs) in the first morning void urine, serum Cr levels, eGFR, urinary L-FABP levels and office BP. The secondary end points also include the estimated 24-h urinary sodium $(\mathrm{Na})$ excretion, plasma aldosterone concentration, urinary aldosterone and cerebro-cardiovascular events. We will evaluate whether the estimated urinary $\mathrm{Na}$ excretion and baseline plasma aldosterone correlate to the degree of the antialbuminuric effect of eplerenone. eGFR will be calculated using the 'Modified Diet in Renal Disease (MDRD) formula' modified by the Japanese Society of Nephrology. ${ }^{28}$ Urinary L-FABP will be measured by an enzymelinked immunosorbent assay (ELISA: Human L-FABP Assay Kit-IBL: ImmunoBiological Laboratories, Takasaki, Japan). Urinary Na excretion over a day will be estimated by a previously reported formula.. ${ }^{29,30}$ Cerebro-cardiovascular events include cerebro-cardiovascular death (fatal myocardial infarction, fatal heart failure, sudden death, fatal stroke and other cardiovascular deaths) and hospitalization due to cerebro-cardiovascular disease (nonfatal myocardial infarction, angina, heart failure, cerebral bleeding, cerebral infarction and transient cerebral ischemic attack).

Other end points. The safety of the eplerenone treatment will be determined by measuring serum potassium $(\mathrm{K})$ changes and adverse events.

\section{Study design}

The double-blinded, randomized, placebo-controlled EVALUATE trial will compare the changes in urinary albumin/Cr ratio of eplerenone- and placebo-treated, hypertensive, RAS inhibitor-treated patients with albuminuria (Figure 1). During each initial screening visit, written informed patient consent will be obtained, interim registration will be performed and all examinations except for urinalysis will be conducted to evaluate patient eligibility, and three urine-sampling kit sets (Uro Catch II, Atleta, Osaka, Japan) for sampling the first morning void urine will be provided. On the second visit, the patient will bring these urine samples. After confirming patient eligibility, each patient will be officially registered and randomly allocated into a $50-\mathrm{mg} \mathrm{day}^{-1}$ eplerenone group or a placebo group. The following factors will be used for stratified randomization: urinary albumin/Cr ratio $\left(<300 \mathrm{mg}, \geqslant 300 \mathrm{mg} \mathrm{g}^{-1}\right)$; systolic BP $(<140 \mathrm{~mm} \mathrm{Hg}$ and $\geqslant 140 \mathrm{~mm} \mathrm{Hg})$; and usage of a particular RAS inhibitor 


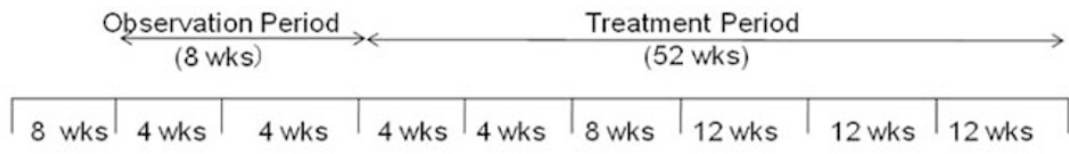

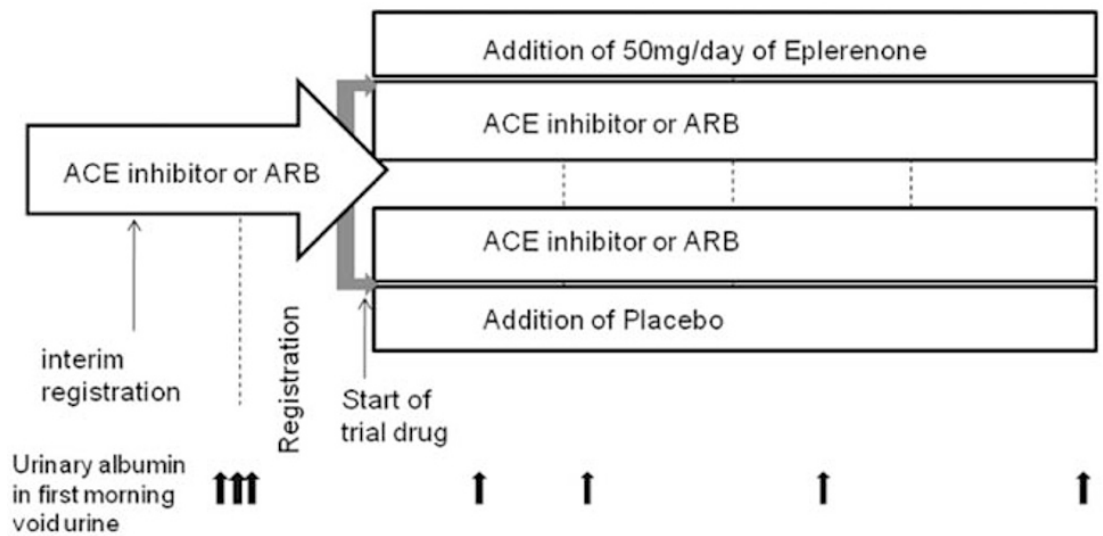

Figure 1 Design of the Eplerenone combination Versus conventional Agents to Lower blood pressure on Urinary Antialbuminuric Treatment Effect (EVALUATE) Trial. ACE inhibitor: angiotensin converting enzyme inhibitor; ARB: angiotensin receptor blocker; wks: weeks. During the trial period, if blood pressure (BP) does not reach $<130 / 80 \mathrm{~mm} \mathrm{Hg}$, an antihypertensive drug other than mineralocorticoid receptor antagonists and renin-angiotensin system inhibitors will be added.

class (ACE inhibitor, ARB orACE inhibitor plus ARB). Thereafter, eplerenone or placebo (both blinded by encapsulation (DBcaps: Capsugel Japan, Sagamihara, Japan)) will be started (together with the RAS inhibitors that will have been given for more than 16 weeks). To ensure that any placebo-treated patients who develop high BP and any eplerenone-treated patients who develop hyperkalemia are promptly treated, the BP and serum $\mathrm{K}$ levels of all patients will be assessed 4 weeks after the trial drug is started. Thereafter, the patients will visit the clinic 8, 16, 28, 40 and 52 weeks after commencing treatment. All prior antihypertensive therapies will continue. If BP does not drop to $<130 /$ $80 \mathrm{~mm} \mathrm{Hg}$, an antihypertensive drug other than MR antagonists and RAS inhibitors will be added.

\section{Study subjects}

Patients to be enrolled in the EVALUATE trial will be hypertensive RAS inhibitor-treated patients with albuminuria who satisfy the following inclusion and exclusion criteria.

Inclusion criteria. The inclusion criteria are: age $\geqslant 20$ year and $<80$ year; outpatient systolic BP $\geqslant 130$ and $<180 \mathrm{~mm} \mathrm{Hg}$ and/or diastolic BP $\geqslant 80$ and $<100 \mathrm{~mm} \mathrm{Hg}$; pretreatment urinary albumin/Cr ratio in the first morning void urine (an average of three continuously measured values) $\geqslant 30$ and $<600 \mathrm{mgg}^{-1}$; eGFR $\geqslant 50 \mathrm{ml} \mathrm{min}^{-1} 1.73 \mathrm{~m}^{-2}$; ACE inhibitor and/or ARB administered for $\geqslant 8$ weeks at interim registration.

Exclusion criteria. Hypertensive emergencies that require intravenous administration of antihypertensives; serum $\mathrm{K} \geqslant 5.0 \mathrm{mEql}^{-1}$; diabetes (fasting blood

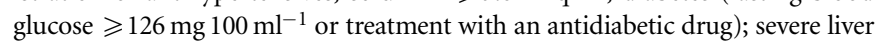
damage (Child-Pugh Score: class C); severe heart failure (New York Heart Association (NYHA) class $\geqslant \mathrm{III}$ ); severe arrhythmia (frequent ventricular or atrial extrasystoles, prolonged ventricular tachycardia, atrial tachyrhythmia with severe tachycardia, atrial fibrillation or flutter with severe tachycardia, sick sinus syndrome with severe bradycardia, atrio-ventricular block with severe bradycardia); angina; myocardial infarction and cerebrovascular disease if they occurred $<6$ months before the interim registration; pregnancy, possibility of pregnancy, desire to become pregnant; a past history of severe side effects from MR antagonists, ACE inhibitors or ARBs; an MR antagonist administered $<8$ weeks before the interim registration; contraindicated drugs, including adrenocorticosteroidal drugs, immunosuppressants, K-sparing diuretics,
$\mathrm{K}$ supplementation, itraconazole, ritonavir and nelfinavir; treatment for $>2$ weeks with non-steroid anti-inflammatory drugs (NSAIDs).

\section{Statistical considerations and study size}

Sample size determination. As RAS-inhibitor treatment of hypertensive patients with albuminuria decreases urinary albumin levels by $45 \%$, whereas RAS inhibitor plus eplerenone decreases it by $74 \%,{ }^{31}$ it is estimated that eplerenone will decrease urinary albumin levels of RAS inhibitor-treated patients by $30 \%$ (that is, an effect size of $30 \%$ ). In addition, estimates are within a s.d. value of the albumin/Cr ratio in the first morning void urine of $90 \%$, a statistical power of $80 \%$, and a dropout rate of $10 \%$. Thus, to compare the eplerenone and placebo groups at a two-sided overall significance level of $5 \%$ with regard to the primary efficacy end point, 340 patients ( 170 patients for each group) will be required.

Statistical analysis. For the efficacy end point, the primary analysis will be carried out on the intent-to-treat (ITT) population (that is, all randomized patients, regardless of patient compliance, actual administration of the trial drug or premature trial drug discontinuation), not including patients deemed ineligible (see eligibility criteria above) or who never take the trial drug. In terms of the safety end point, all patients who take the trial drug will be analyzed.

The eplerenone and placebo groups will be compared in terms of the percentage change in the urinary albumin/Cr ratio after 12 months of treatment relative to the pretreatment ratio by using an unpaired $t$-test at a two-sided significance level of $5 \%$. If necessary, the data will be adjusted by important background factors, including gender, age, $\mathrm{BP}$ response to the treatment, eGFR level, urinary L-FABP levels and urinary $\mathrm{Na}$ excretion. In addition, analysis with a linear model will be performed for percent changes in the urinary albumin/Cr ratio from the pretreatment period to each point of treatment, in the absolute value of urinary albumin/Cr ratio, and for their timedependent changes. The absolute values and changes over time of the following parameters will also be analyzed: urinary L-FABP level, office BP, eGFR level, estimated $\mathrm{Na}$ excretion over a day and plasma aldosterone concentration. The groups will also be compared in terms of the rate of CKD stage progression, allcause mortality and frequency of cardiovascular events (cerebro-cardiovascular death (fatal myocardial infarction, fatal heart failure, sudden death, fatal stroke and other cardiovascular death) and hospitalization due to cerebro-cardiovascular events (non-fatal myocardial infarction, angina, heart failure, cerebral 
bleeding, cerebral infarction and transient cerebral ischemic attack)). To evaluate trial drug safety, serum $\mathrm{K}$ levels will be analyzed. The two groups will also be compared in terms of the frequency of adverse events and the rate of dropout due to adverse events.

\section{Registration and ethics principles}

The EVALUATE study has been registered at the University Hospital Medical Information Network-Clinical Trials Registry (UMIN-CTR) under the trial identification number UMIN000001803. It has been approved by the Institutional Review Boards of the University of Tokyo Clinical Research Center (reference number P2008028-11X) and other hospitals, and it will be conducted in accordance with the Declaration of Helsinki Principles. Consequently, written informed consent will be obtained after patients receive an oral and written explanation of the trial from the attending physician.

\begin{abstract}
Management of the study
The organization and members of each committee of the EVALUATE trial are shown in the Appendix A. The Principal Study Coordinators (Professor Fujita (responsible for trial implementation) and Professor Yamada (responsible for trial fund management)) and the Steering Committee will oversee and are responsible for conducting the trial, including reviewing and implementing recommendations from the Independent Data Monitoring and Safety Committee, protocol changes and premature study termination. The Steering Committee will be blinded to the treatment assignments and will take responsibility for publications arising from the trial. The Protocol Committee is responsible for study design and protocol development as well as any changes. The Coordinating Committee is responsible for organizing the committees and secretarial work. The Representative for Trial Drug Preparation ensures eplerenone and placebo encapsulation (DBcaps: Capsugel Japan; performed in the prescription laboratory of the Pharmaceutical Department of the Tokyo University Hospital). The Representative for the Trial Drug Masking ensures eplerenone and placebo blinding. The Representative for the Trial Drug Management is responsible for the trial drug storage and delivery to the trial site. The Data Center is responsible for data management (including operating the Internet system dealing with patient registry and enrolling data and handling the individual case safety reports of serious adverse events. The Data Monitoring and Safety Committee will assess safety and end points, such as progression of CKD and CVD, evaluate adverse events, oversee patient welfare, review trial data at specified intervals and make recommendations to the Steering Committee if any problems arise (for example, serious adverse events). The latter two committees do not include investigators in the study. All study investigators are experienced clinical trialists without any conflict of interest with respect to the sponsor (Pfizer Japan, Tokyo, Japan).
\end{abstract}

\section{DISCUSSION}

The EVALUATE study asks whether the MR antagonist, eplerenone, has an antialbuminuric effect in RAS inhibitor-treated hypertensive patients at an early stage of CKD (mild-moderate albuminuria and eGFR $\geqslant 50 \mathrm{ml} \mathrm{min}^{-1} 1.73 \mathrm{~m}^{-2}$ ). Many patients in the early stage of CKD eventually exhibit disease progression, at which point renoprotective medical procedures become less effective and the patient develops ESKD. Thus, proper CKD management requires effective therapeutic strategies that reduce its progression in its early stages. Although almost all CKD patients require more than one antihypertensive to control BP, only weak evidence supports the idea that CCBs or diuretics should be the second-line antihypertensive agents for RAS inhibitor-treated CKD patients with uncontrolled BP. ${ }^{1}$ Moreover, a recent meta-analysis revealed that the dihydropyridine CCBs do not decrease urinary protein levels. ${ }^{32}$ In addition, the Ramipril Efficacy In Nephropath-2 (REIN-2) study ${ }^{4}$ showed that, in patients with nondiabetic $\mathrm{CKD}$, strict $\mathrm{BP}$ reduction (to $<130 / 80 \mathrm{~mm} \mathrm{Hg}$ ) by adding the $\mathrm{CCB}$, felodipine, to the ACE inhibitor, ramipril, did not result in superior renoprotection when compared with ramipril treatment alone, which only achieved a moderate BP response (diastolic BP
$<90 \mathrm{~mm} \mathrm{Hg}$ ). The GaUging Albuminuria Reduction with Lotrel in Diabetic patients with hypertension (GUARD) study of hypertensive type 2 diabetic patients ${ }^{5}$ indicated that, relative to the combination of the ACE inhibitor, benazepril, and the CCB, amlodipine, the combination of benazepril and the diuretic, hydrochlorothiazide, was superior in reducing urinary albumin levels; however, the latter combination was also associated with significantly lower eGFR level. Thus, there is little definitive evidence supporting the rationale that the second-line drugs used to treat RAS inhibitor-treated CKD patients with uncontrolled BP should be CCBs, diuretics or other drugs. By contrast, animal and small-sized clinical studies have recently revealed that $\mathrm{MR}$ antagonists have a renoprotective effect that might be superior to RAS inhibitors. ${ }^{6-8,12,13,15,18,19}$ Thus, the EVALUATE trial may indicate that MR antagonists should be used as second-line antihypertensive agent for patients with CKD.

One potential limitation of MR antagonists as second-line drugs for CKD patients is their $\mathrm{K}$-sparing effect. This effect may be disadvantageous for patients with CKD, especially when the MR antagonist is administered in combination with a RAS inhibitor, because both renal dysfunction and RAS inhibitors can increase serum K level. However, a study of diabetic nephropathy patients with normal renal function showed that, compared with the ACE inhibitor, enarapril, alone, co-administration of a relatively low dose $\left(50-100 \mathrm{mg}\right.$ day $\left.^{-1}\right)$ of eplerenone with enarapril significantly reduces albuminuria without significantly increasing hyperkalemia. ${ }^{19}$ Thus, in the EVALUATE trial, we will examine whether the low dose $\left(50 \mathrm{mg} \mathrm{day}^{-1}\right)$ of eplerenone can exert an antialbuminuric effect in patients at an early stage of CKD (that is, patients with albuminuria and eGFR $\geqslant 50 \mathrm{ml} \mathrm{min}^{-1} 1.73 \mathrm{~m}^{-2}$ ) without concomitantly increasing hyperkalemia.

We will also focus on salt intake in patients because MR blockade could be advantageous for CKD patients with a high-salt diet for two reasons. First, the MR antagonists have a greater renoprotective effect in salt-repleted rather than salt-depleted conditions because MR activation seems to have an important mediating role in the renal damage induced by excess salt. ${ }^{6,20-24}$ Second, hyperkalemia may occur less frequently in patients with a high salt diet than a low salt diet, because salt loading decreases serum $\mathrm{K}$ level. ${ }^{33}$ Moreover, animal studies, including our own, ${ }^{34,35}$ suggest that a mild increase in serum $\mathrm{K}$ level may promote organ protection in relatively $\mathrm{K}$-depleted CKD patients with a high salt intake.

The EVALUATE trial has several limitations. Our inclusion criteria include patients who have an eGFR $\geqslant 50 \mathrm{ml} \mathrm{min}^{-1} 1.73 \mathrm{~m}^{-2}$ and are not diabetic. Nevertheless, worthwhile information can be elicited from this trial, because it will elucidate whether aldosterone blockade is a useful strategy to treat patients with early stage CKD. Another putative study limitation is that the primary end point is urinary albumin. However, this end point was chosen over renal function or CVD, because these end points would necessitate a much longer trial period.

In conclusion, the EVALUATE study may show that a low dose of eplerenone effectively decreases urinary albumin levels in RAS inhibitor-treated non-diabetic hypertensive patients with albuminuria and eGFR $\geqslant 50 \mathrm{ml} \mathrm{min}^{-1} 1.73 \mathrm{~m}^{-2}$ without concomitantly inducing hyperkalemia. The Japanese people in particular, many of whom consume a high salt diet, are expected to benefit from treatment with MR antagonists. However, because most people in the world consume more salt than is ideal, we anticipate that the EVALUATE trial results can also be extrapolated to populations worldwide.

\section{CONFLICT OF INTEREST}

The authors declare no conflict of interest. 


\section{ACKNOWLEDGEMENTS}

This study is financially supported by Pfizer Japan.

1 Ogihara T, Kikuchi K, Matsuoka H, Fujita T, Higaki J, Horiuchi M, Imai Y, Imaizumi T, Ito S, Iwao H, Kario K, Kawano Y, Kim-Mitsuyama S, Kimura G, Matsubara H, Matsuura H, Naruse M, Saito I, Shimada K, Shimamoto K, Suzuki H, Takishita S, Tanahashi N, Tsuchihashi T, Uchiyama M, Ueda S, Ueshima H, Umemura S, Ishimitsu T, Rakugi H, The Japanese Society of Hypertension Committee. The Japanese Society of Hypertension Guidelines for the Management of Hypertension (JSH 2009). Hypertens Res 2009; 32: 3-107.

2 Mancia G, De Backer G, Dominiczak A, Cifkova R, Fagard R, Germano G, Grassi G, Heagerty AM, Kjeldsen SE, Laurent S, Narkiewicz K, Ruilope L, Rynkiewicz A, Schmieder RE, Boudier HA, Zanchetti A, Vahanian A, Camm J, De Caterina R, Dean V, Dickstein K, Filippatos G, Funck-Brentano C, Hellemans I, Kristensen SD, McGregor K, Sechtem U, Silber S, Tendera M, Widimsky P, Zamorano JL, Erdine S, Kiowski W, Agabiti-Rosei E, Ambrosioni E, Lindholm LH, Viigimaa M, Adamopoulos S, Agabiti-Rosei E, Ambrosioni E, Bertomeu V, Clement D, Erdine S, Farsang C, Gaita D, Lip G, Mallion JM, Manolis AJ, Nilsson PM, O'Brien E, Ponikowski P, Redon J, Ruschitzka F, Tamargo J, van Zwieten P, Waeber B, Williams B, Management of Arterial Hypertension of the European Society of Hypertension; European Society of Cardiology. 2007 Guidelines for the Management of Arterial Hypertension. The Task Force for the Management of Arterial Hypertension of the European Society of Hypertension (ESH) and of the European Society of Cardiology (ESC). J Hypertens 2007: 25: 1105-1187.

3 Chobanian AV, Bakris GL, Black HR, Cushman WC, Green LA, Izzo Jr JL, Jones DW, Materson BJ, Oparil S, Wright Jr JT, Roccella EJ, Joint National Committee on Prevention, Detection, Evaluation, and Treatment of High Blood Pressure. National Heart, Lung, and Blood Institute; National High Blood Pressure Education Program Coordinating Committee. Seventh report of the Joint National Committee on Prevention, Detection, Evaluation, and Treatment of High Blood Pressure. Hypertension 2003; 42: 1206-1252.

4 Ruggenenti P, Perna A, Loriga G, Ganeva M, Ene-lordache B, Turturro M, Lesti M, Perticucci E, Chakarski IN, Leonardis D, Garini G, Sessa A, Basile C, Alpa M, Scanziani R, Sorba G, Zoccali C, Remuzzi D, REIN-2 Study Group. Blood-pressure control for renoprotection in patients with non-diabetic chronic renal disease (REIN-2): multicentre, randomised controlled trial. Lancet 2005; 365: 939-946.

5 Bakris GL, Toto RD, McCullough PA, Rocha R, Purkayastha D, Davis P, GUARD (Gauging Albuminuria Reduction With Lotrel in Diabetic Patients With Hypertension) Study Investigators. Effects of different ACE inhibitor combinations on albuminuria: results of the GUARD study. Kidney Int 2008; 73: 1303-1309.

6 Shibata S, Nagase M, Yoshida S, Kawachi H, Fujita T. Podocyte as the target for aldosterone: roles of oxidative stress and Sgk1. Hypertension 2007; 49: 355-364.

7 Nagase M, Yoshida S, Shibata S, Nagase T, Gotoda T, Ando K, Fujita T. Enhanced aldosterone signaling in the early nephropathy of rats with metabolic syndrome: possible contribution of fat-derived factors. J Am Soc Nephrol 2006; 17: 3438-3446.

8 Nagase M, Shibata S, Yoshida S, Nagase T, Gotoda T, Fujita T. Podocyte injury underlies the glomerulopathy of Dahl salt-hypertensive rats and is reversed by aldosterone blocker. Hypertension 2006; 47: 1084-1093.

9 Farjah M, Roxas BP, Geenen DL, Danziger RS. Dietary salt regulates renal SGK1 abundance: relevance to salt sensitivity in the Dahl rat. Hypertension 2003; 41: 874-878.

10 Nagase M, Matsui H, Shibata S, Gotoda T, Fujita T. Salt-induced nephropathy in obese spontaneously hypertensive rats via paradoxical activation of the mineralocorticoid receptor: role of oxidative stress. Hypertension 2007; 50: 877-883.

11 Shibata S, Nagase M, Yoshida S, Kawarazaki W, Kurihara H, Tanaka H, Miyoshi J, Takai Y, Fujita T. Modification of mineralocorticoid receptor function by Rac1 GTPase: implication in proteinuric kidney disease. Nat Med 2008; 14: 1370-1376.

12 Williams GH, Burgess E, Kolloch RE, Ruilope LM, Niegowska J, Kipnes MS, Roniker B, Patrick JL, Krause SL. Efficacy of eplerenone versus enalapril as monotherapy in systemic hypertension. Am J Cardiol 2004; 93: 990-996.

13 Onozato ML, Tojo A, Kobayashi N, Goto A, Matsuoka H, Fujita T. Dual blockade of aldosterone and angiotensin II additively suppresses TGF-beta and NADPH oxidase in the hypertensive kidney. Nephrol Dial Transplant 2007; 22: 1314-1322.

14 Rocha R, Chander PN, Zuckerman A, Stier Jr CT. Role of aldosterone in renal vascular injury in stroke-prone hypertensive rats. Hypertension 1999; 33: 232-237.

15 Sato A, Hayashi K, Naruse M, Saruta T. Effectiveness of aldosterone blockade in patients with diabetic nephropathy. Hypertension 2003; 41: 64-68.

16 Pitt B, Zannad F, Remme WJ, Cody R, Castaigne A, Prez A, Palensky J, Wittes J, Randomized Aldactone Evaluation Study Investigators. The effect of spironolactone on morbidity and mortality in patients with severe heart failure. N Eng/ J Med 1999; 341: 709-717.

17 Pitt B, Remme W, Zannad F, Neaton J, Martinez F, Roniker B, Bittman R, Hurley S, Kleiman J, Gatlin M, Eplerenone Post-Acute Myocardial Infarction Heart Failure Efficacy and Survival Study Investigators. Eplerenone, a selective aldosterone blocker, in patients with left ventricular dysfunction after myocardial infarction. N Engl J Med 2003; 348: 1309-1321.
18 White WB, Duprez D, St Hillaire R, Krause S, Roniker B, Kuse-Hamilton J, Weber MA. Effects of the selective aldosterone blocker eplerenone versus the calcium antagonist amlodipine in systolic hypertension. Hypertension 2003; 41: 1021-1026.

19 Epstein M, Williams GH, Weinberger M, Lewin A, Krause S, Mukherjee R, Patni R, Beckerman B. Selective aldosterone blockade with eplerenone reduces albuminuria in patients with type 2 diabetes. Clin J Am Soc Nephrol 2006; 1: 940-951.

20 Hartner A, Porst M, Gauer S, Proels F, Veelken R, Hilqers KF. Glomerular osteopontin expression and macrophage infiltration in glomerulosclerosis of DOCA-salt rats. Am J Kidney Dis 2001; 38: 153-164.

21 Blasi ER, Rocha R, Rudolph AE, Blomme EA, Polly ML, McMahon EG. Aldosterone/salt induces renal inflammation and fibrosis in hypertensive rats. Kidney Int 2003; 63: 1791-1800.

22 Pimenta E, Gaddam KK, Pratt-Ubunama MN, Nishizaka MK, Aban I, Oparil S, Calhoun DA. Relation of dietary salt and aldosterone to urinary protein excretion in subjects with resistant hypertension. Hypertension 2008; 51: 339-344.

23 Sato A, Saruta T. Aldosterone-induced organ damage: plasma aldosterone level and inappropriate salt status. Hypertens Res 2004; 27: 303-310.

24 Pimenta E, Calhoun DA. Aldosterone, dietary salt, and renal disease. Hypertension 2006; 48: 209-210.

25 Brown IJ, Izoulaki I, Candeias V, Elliott P. Salt intakes around the world: implications for public health. Int J Epidemiol 2009; 38: 791-813.

26 Yamamoto T, Noiri E, Ono Y, Doi K, Negishi K, Kamijo A, Kimura K, Fujita T, Kinukawa T, Taniguchi H, Nakamura K, Goto M, Shinozaki N, Ohshima S, Sugaya T. Renal L-type fatty acid-binding protein in acute ischemic injury. J Am Soc Nephrol 2007; 18: 2894-2902.

27 Kamijo A, Sugaya T, Hikawa A, Yamanouchi M, Hirata Y, Ishimitsu T, Numabe A, Takagi M, Hayakawa H, Tabei F, Sugimoto T, Mise N, Kimura K. Clinical evaluation of urinary excretion of liver-type fatty acid-binding protein as a marker for the monitoring of chronic kidney disease: A multicenter trial. J Lab Clin Med 2005; 145: 125-133.

28 Matsuo S, Imai E, Horio M, Yasuda Y, Tomita K, Nitta K, Yamagata K, Tomino Y, Yokoyama $\mathrm{H}$, Hishida A, Collaborators developing the Japanese equation for estimated GFR. Revised equations for estimated GFR from serum creatinine in Japan. Am J Kidney Dis 2009; 53: 982-992.

29 Kawano Y, Tsuchihashi T, Matsuura H, Ando K, Fujita T, Ueshima H, Working Group for Dietary Salt Reduction of the Japanese Society of Hypertension. Report of the Working Group for Dietary Salt Reduction of the Japanese Society of Hypertension: (2) Assessment of salt intake in the management of hypertension. Hypertens Res 2007; 30: 887-893.

30 Kawasaki T, Itoh K, Uezono K, Sasaki H. A simple method for estimating $24 \mathrm{~h}$ urinary sodium and potassium excretion from second morning voiding urine specimen in adults. Clin Exp Pharmacol Physiol 1993; 20: 7-14.

31 Hollenberg NK. Aldosterone in the development and progression of renal injury. Kidney Int 2004; 66: 1-9.

32 Bakris GL, Weir M, Secic M, Campbell B, Weis-McNulty A. Differential effects of calcium antagonist subclasses on markers of nephropathy progression. Kidney Int 2004; 65: 1991-2002.

33 Fujita T, Ando K, Ogata E. Systemic and regional hemodynamics in patients with salt-sensitive hypertension. Hypertension 1990; 16: 235-244.

34 Matsui H, Shimosawa T, Uetake Y, Wang H, Ogura S, Kaneko T, Liu J, Ando K, Fujita T. Protective effect of potassium against the hypertensive cardiac dysfunction. Hypertension 2006; 48: 225-231

35 Kido M, Ando K, Onozato ML, Tojo A, Yoshikawa M, Ogita T, Fujita T. Protective effect of dietary potassium against the vascular injury in salt-sensitive hypertension. Hypertension 2008; 51: 225-231.

\section{APPENDIX A}

EVALUATE (Eplerenone combination Versus conventional Agents to Lower blood pressure on Urinary Antialbuminuric Treatment

\section{Effect) study group}

Principal Study Coordinators

Toshiro Fujita, Department of Nephrology and Endocrinology, Faculty of Medicine, University of Tokyo, Tokyo, Japan

Akira Yamada, First Department of Internal Medicine, Kyorin University School of Medicine, Tokyo, Japan

Steering Committee

Toshiro Fujita, Department of Nephrology and Endocrinology, Faculty of Medicine, University of Tokyo, Tokyo, Japan Akira Yamada, First Department of Internal Medicine, Kyorin University School of Medicine, Tokyo, Japan

Kenjiro Kimura, Department of Nephrology and Hypertension, St Marianna University School of Medicine, Kawasaki, Japan Shunya Uchida, Division of Nephrology, Department of Internal Medicine, Teikyo University School of Medicine, Tokyo, Japan 


\section{Protocol Committee}

Akira Yamada, First Department of Internal Medicine, Kyorin University School of Medicine, Tokyo, Japan

Katsuyuki Ando, Department of Nephrology and Endocrinology, Faculty of Medicine, University of Tokyo, Tokyo, Japan

Tetsuo Nishikawa, Department of Medicine, Yokohama Rosai Hospital, Yokohama, Japan

Atsuhisa Sato. Department of Internal Medicine, International University of Health and Welfare Mita Hospital, Tokyo, Japan

Miki Nagase, Department of Nephrology and Endocrinology, Faculty of Medicine, University of Tokyo, Tokyo, Japan

Coordinating Committee

Toshiro Fujita, Department of Nephrology and Endocrinology, Faculty of Medicine, University of Tokyo, Tokyo, Japan

Katsuyuki Ando, Department of Nephrology and Endocrinology, Faculty of Medicine, University of Tokyo, Tokyo, Japan

George Seki, Department of Nephrology and Endocrinology, Faculty of Medicine, University of Tokyo, Tokyo, Japan

Akihiro Tojo, Department of Nephrology and Endocrinology, Faculty of Medicine, University of Tokyo, Tokyo, Japan

Masaomi Nangaku, Department of Nephrology and Endocrinology, Faculty of Medicine, University of Tokyo, Tokyo, Japan

Tatsuo Shimosawa, Department of Nephrology and Endocrinology, Faculty of Medicine, University of Tokyo, Tokyo, Japan

Shinya Kaname, First Department of Internal Medicine, Kyorin University School of Medicine, Tokyo, Japan

Yugo Shibagaki, Department of Nephrology and Hypertension, St Marianna University School of Medicine, Kawasaki, Japan

Representatives for the Trial Drug Preparation

Hiroshi Suzuki, Department of Pharmacy, University of Tokyo Hospital, Faculty of Medicine, University of Tokyo, Tokyo, Japan Katsuyoshi Nakajima, Department of Pharmacy, University of Tokyo Hospital, Faculty of Medicine, University of Tokyo, Tokyo, Japan
Yoshitugu Yanagihara, Department of Pharmacy, University of Tokyo Hospital, Faculty of Medicine, University of Tokyo, Tokyo, Japan

Representatives for the Trial Drug Masking and the Management Yoshihiro Arakawa, University of Tokyo Clinical Research Center, University of Tokyo Hospital, Faculty of Medicine, University of Tokyo, Tokyo, Japan

Atsushi Aoki, University of Tokyo Clinical Research Center, University of Tokyo Hospital, Faculty of Medicine, University of Tokyo, Tokyo, Japan

Data Center

Takuhiro Yamaguchi, Department of Clinical Trial Data Management, Faculty of Medicine, University of Tokyo, Tokyo, Japan

Hiroshi Ohtsu, Department of Clinical Trial Data Management, Faculty of Medicine, University of Tokyo, Tokyo, Japan

Takahiro Kiuchi, University Hospital Medical Information Network Center, University of Tokyo Hospital, Faculty of Medicine, University of Tokyo, Tokyo, Japan

Hiromu Nishiuchi, University Hospital Medical Information Network Center, University of Tokyo Hospital, Faculty of Medicine, University of Tokyo, Tokyo, Japan

Kiyoshi Kubota, Department of Pharmacoepidemiology, Faculty of Medicine, University of Tokyo, Tokyo, Japan

Data Monitoring and Safety Committee

Tsutomu Yamazaki, Department of Clinical Epidemiology and Systems, Faculty of Medicine, University of Tokyo, Tokyo, Japan

Shinobu Tatsunami, Unit of Medical Statistics, Faculty of Medical Education and Culture, St Marianna University School of Medicine, Kawasaki, Japan

Toshiyuki Imasawa, Department of Internal Medicine, Division of Immunopathology, Clinical Research Center, Chiba-East National Hospital, Chiba, Japan 